\title{
Psyllium Husk
}

National Cancer Institute

\section{Source}

National Cancer Institute. Psyllium Husk. NCI Thesaurus. Code C29250.

The husk of the dried ripe seeds of the Plantago psyllium, P. Indica, P. Ovata, and P. Arenaria plants with laxative and cholesterol lowering activities. The husks contain mucilage that swells on exposure to water and provides an indigestible mucilaginous mass in the intestines, thereby causing lubrication, contraction of the smooth muscles of the intestinal walls, and thus stimulating bowel movement. Psyllium husk is rich in soluble fiber and has a cholesterol-lowering effect. 